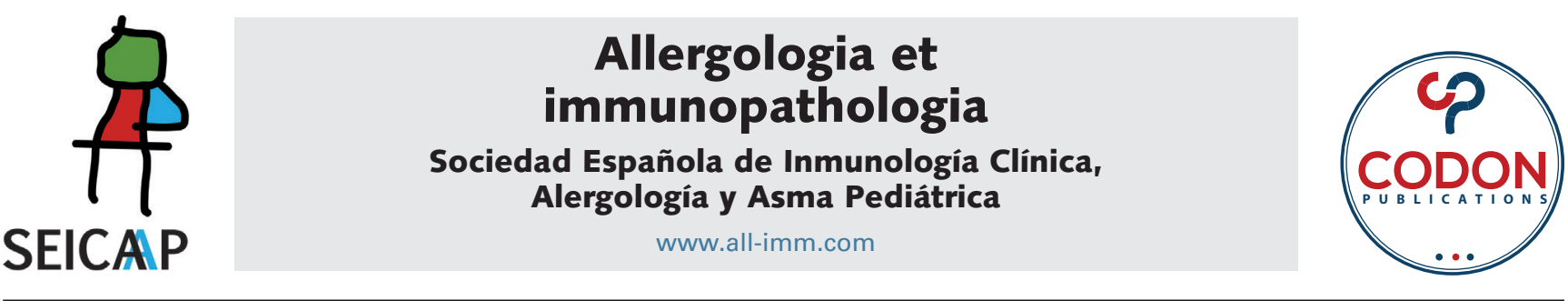

ORIGINAL ARTICLE

\title{
Allergen-specific subcutaneous immunotherapy-pain evaluation in pediatric age
}

\author{
Cristiana Ferreira ${ }^{a *}$, Isabel Rezende ${ }^{\mathrm{b}}$, Arminda Guilherme $^{\mathrm{a}}$, Inês Lopes ${ }^{\mathrm{a}}$
}

${ }^{a}$ Allergy and Clinical Immunology Department, Centro Hospitalar Vila Nova de Gaia/Espinho, EPE, Vila Nova de Gaia, Portugal ${ }^{b}$ Allergy and Clinical Immunology Department, Centro Hospitalar do Porto, Porto, Portugal

Received 22 March 2020; Accepted 27 September 2020

Available online 2 January 2021

\section{KEYWORDS \\ pain; \\ allergen-specific \\ subcutaneous \\ immunotherapy; \\ allergic pediatrics \\ patients}

\begin{abstract}
Background: Allergen-specific immunotherapy is a potentially disease-modifying therapy that is effective for the treatment of patients with allergic diseases. Although the pain caused by the administration of subcutaneous immunotherapy with allergens (SCITA) is considered to be minimal, no studies assessing that pain for the treatment of only pediatric patients have been reported.

Objectives: This research aimed to evaluate the pain associated with SCITA for pediatric patients followed at our Immunoallergology Department.

Methods: During four consecutive weeks, the nurse who administered the injection completed a questionnaire recording the child's assessment of the pain associated with SCITA; these questionnaires were randomized before any analyses were done. Two different pain evaluation scales were used, with the choice of scale being determined based on the child's age: the self-reporting faces scale (score: $0-10 ; 5$ to 8 years old) and the numeric scale (score: $0-10 ;>8$ years old). Demographic and clinical data, as well as any adverse reactions, were documented. Results: We included 46 pediatric patients (mean age: $12.3 \pm 2.6$ years; $69.5 \%$ male), most of whom were suffering from rhinitis/rhinoconjunctivitis and undergoing subcutaneous immunotherapy with mites. Seven local adverse reactions were recorded, and all were mild. Ten patients did not mention any pain associated with SCITA. Of the 36 patients who mentioned some pain, 33 mentioned mild pain (scores between 1 and 3); only three mentioned moderate pain (scores between 4 and 6). For both scales, the median score obtained was 1 . The maximum pain reported had a score of 6 . No significant differences were observed between different groups of patients.

Conclusions: In this study, SCITA was shown to be a mildly painful procedure that is associated with only a few local reactions. Therefore, SCITA should be considered as a safe option for the treatment of most pediatric patients suffering from allergies.

(c) 2021 Codon Publications. Published by Codon Publications.
\end{abstract}

*Corresponding author: Cristiana Ferreira. Allergy and Clinical Immunology Department, Centro Hospitalar Vila Nova de Gaia/Espinho, EPE, Vila Nova de Gaia, Portugal. Email address: cristianascferreira@gmail.com 


\section{Introduction}

The prevalence of allergic respiratory disease continues to rise, and effective multimodal strategies are required to achieve optimal control of symptoms and prevent progression of the atopic condition. In a therapeutic field dominated by pharmacotherapy, allergen immunotherapy, with its disease-modifying traits and unique mechanisms of action, including the generation of Treg cells and the induction of protective antibodies, may be an underused option that merits cautious consideration for the management of allergic respiratory diseases in suitable patients. Despite the significant heterogeneity in study design, multiple meta-analyses have supported the efficacy and safety of allergen-specific immunotherapy (SIT) for treating patients with allergic respiratory diseases. SIT involves the repeated subcutaneous (SCIT) or sublingual (SLIT) administration over a period of 3 to 5 years of allergenic extracts to atopic individuals with allergic respiratory diseases, including asthma and allergic rhinitis. ${ }^{1}$ Pending definitive comparative trials, the choice between SCIT and SLIT will most likely be determined based on the local availability of approved SCIT and SLIT products and on personal (patient) preference. ${ }^{2,3}$ Although the pain caused by the administration of subcutaneous immunotherapy with allergens (SCITA) is considered to be minimal, no studies assessing that pain in only pediatric patients have been reported.

According to the International Association for the Study of Pain, "Pain is an unpleasant sensory and emotional experience associated with actual or potential tissue damage". ${ }^{4}$

Pain is considered as the fifth vital sign, presupposing a systematic assessment and a recording of its intensity by using evaluation tools. Perception of pain in pediatric patients is complex and unique due to factors inherent in the technique and the injected material, as well as individual factors such as the child's age, developmental level, cognitive and communication skills, and past experiences with pain. ${ }^{5}$ Thus, accurate pain measurements in children are difficult. Three main methods are currently used to measure pain intensity: self-reporting, behavioral measures, and physiological measures. Self-reporting is optimal and the most valid. ${ }^{6}$ Self-assessment of pain involves an evaluation by the person experiencing it and is done using self-assessment or self-report scales. ${ }^{7,8}$

Self-assessment tools are suitable for children older than 5 years of age. Scales with several levels of pain intensity, such as faces or numeric scales, are used in children capable of performing their own evaluation (self-assessment). The child can use these tools to describe the level of pain experienced during the procedure., ${ }^{7,8}$

Our study aimed to evaluate the pain caused by SCITA in pediatric patients followed at our Immunoallergology Department. The self-assessments given by the pediatric patients were analyzed to determine possible correlations between the intensity of pain and demographic and clinical factors and/or manifested adverse reactions.

\section{Material and methods}

Forty-six pediatric patients (mean age: $12.3 \pm 2.6$ years; $69.5 \%$ male) who were being treated at our Immunoallergology
Department were included in this study. Most of those patients were suffering from rhinitis/rhinoconjunctivitis and were undergoing subcutaneous immunotherapy with mites. The children's parents or guardians gave informed consent for their participation in this study.

The SCITA procedure was performed by a trained nursing team. Disposable 26-G, $1-\mathrm{mL}$ syringes with diameters of $0.45 \mathrm{~mm}$ and lengths of $12.7 \mathrm{~mm}$ were used for the subcutaneous injections, and the SCITA procedure was the same for all patients. Pain evaluation scales were chosen based on the child's age: a self-reporting faces scale (score: 0 to 10) was chosen for children who were 7 or 8 years of age, whereas a numeric scale (score: 0 to 10 ) was chosen for children over the age of 8 years. The pain perception rate (PPR) was evaluated after the injection. For four consecutive weeks, the nurses who had administered the injections completed questionnaires evaluating the intensities of pain associated with the subcutaneous injections of allergen immunotherapy, as reported by the pediatric patients. The following aspects were assessed: demographic data (sex, age, allergic diseases), SCITA data (laboratory, type of extract, dose and site of the administration: left/right arm or both, and initiation date), pain evaluation (none: 0 , mild: $1-3$, moderate: 4-6, or severe: $7-10$ ), and immediate adverse reactions (local/systemic and type: pruritus, erythema, papule, etc.).

Collected data were randomized by the researchers for analysis. Statistical analyses were performed using SPSS 22.0 for Windows ${ }^{\circledR}$ (SPSS Inc., Chicago, USA). Descriptive analyses were performed using absolute frequencies, and the results are reported as means \pm standard deviations or medians and inter-quartile intervals. The differences were tested using the Mann-Whitney U-test for independent samples or the Kruskall-Wallis test.

\section{Results}

Forty-six pediatric patients, aged from 7 to 16 years old, who were receiving SCITA at our Immunoallergology Department were included in this study. Their mean age was $12.28 \pm 2.6$ years and $69.5 \%$ were male. The majority of patients were diagnosed with allergic rhinitis/rhinoconjunctivitis (65.3\%) or concomitant asthma (17.4\%). The demographic data are provided in Table 1. SCITA was

Table1 Demographic and Clinical data

\begin{tabular}{ll}
\hline Gender & $\mathbf{N}(\%)$ \\
\hline Female & $14(30,5 \%)$ \\
Male & $32(69,5 \%)$ \\
Age & \\
Mean \pm DP: $12,28 \pm 2,605(7-16)$ years-old & \\
$>8$ years old $(\mathrm{n}=42,91.30 \%)$ & \\
Allergic diseases & $17(37 \%)$ \\
Rhinitis & $13(28,3 \%)$ \\
Rhinoconjuntivitis & $8(17,4 \%)$ \\
Rhinitis+Asthma & $5(10,8 \%)$ \\
Asthma+Rhinitis+Eczema & $2(4,3 \%)$ \\
Asthma +Rhinoconjuntivitis+Eczema & $1(2,2 \%)$ \\
Asthma & \\
\hline
\end{tabular}


administered to patients by using polymerized extracts purchased from four laboratories. The allergen extracts could be divided into two principal groups: mites and pollens. Doses ranged from 0.20 to $0.5 \mathrm{~mL}$, with most being $0.5 \mathrm{~mL}$ (73.9\%). In $60.9 \%$ of the patients, the SCITA site was the left upper arm, and in 39.1\%, it was the right upper arm. Pain evaluation scales were chosen based on the child's age. The self-assessment scale of faces was used in four children, and the numerical self-assessment was used in 42 children. Ten (21.7\%) patients did not mention having any pain associated with the administration of SCITA (pain 0). Of the 36 patients who mentioned some pain, 33 indicated mild pain (values 1 to 3 ) and three reported moderate pain (values 4 to 6). For both scales, the median and the mean pain intensities were 1 and 1.4, respectively. The maximum pain reported was 6 (Figure 1).

Most of the patients (87.1\%) did not report any adverse reactions following SCITA. Local reactions (12.9\%) corresponding to redness $(n=7)$ and itching $(n=2)$ at the injection site were reported by seven patients. All reactions were considered as mild. No patient presented with a hematoma, and no systemic reactions occurred. Regarding demographic data (gender and age) and clinical data (extract composition, dose, site of injection, and occurrence of local reactions), no statistically significant differences were found between the median values of pain in the different groups. However, in regard to age, younger children (7 or 8 years of age) were found to have a higher median pain $($ score $=2)$ than older children. Extract allergen from laboratory 3, pollen extracts, and a dose of $0.5 \mathrm{~mL}$ were found to be associated with a slightly higher self-reported intensity of pain (median score $=2$ ).

\section{Discussion}

Pediatrics patients are regularly subjected to a variety of medical procedures. Some procedures are more painful and less tolerated than others; as a result, many patients have come to fear medical procedures. ${ }^{9}$ Overall, few studies have investigated pain during medical procedures to

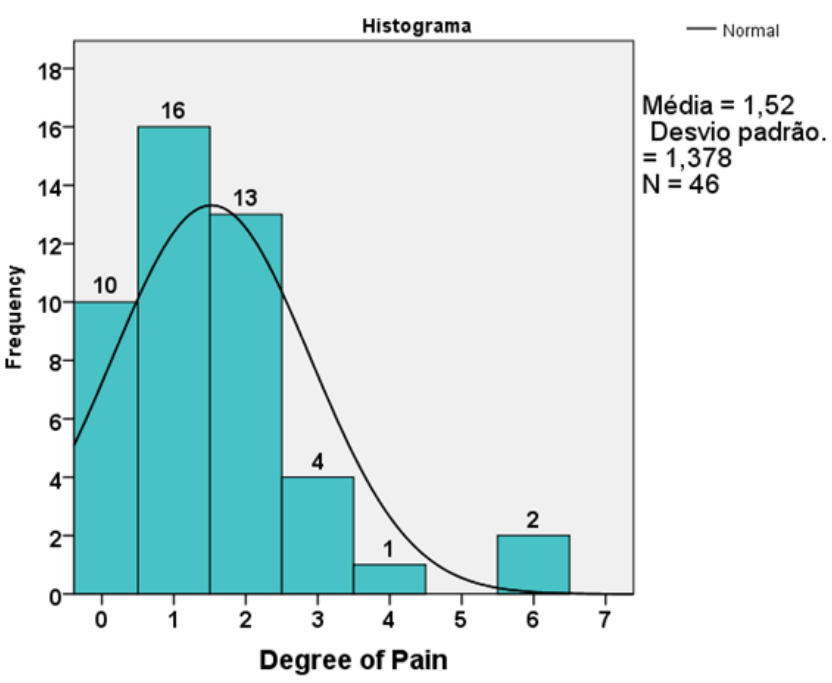

Figure 1 Degree of pain reported after SCITA administration. treat patients with allergies, especially pediatric patients. We describe the results of a prospective analysis of pain scores in young children receiving subcutaneous allergy immunotherapy. In our study, the median score on a scale of 10 for the intensity of pain experienced by pediatric patients undergoing SCITA was 1, indicating mild pain. This low score is most likely due to the experience of the nurses who administered the injections and the materials that were used. Compared to other studies that have measured pain associated with analogous procedures, our participants reported a relatively low amount of pain. Spínola et al. ${ }^{10}$ studied a group of patients with respiratory allergies treated with allergen subcutaneous immunotherapy and found that children had a higher median pain than adults, with a median pain intensity of 2 for children as compared to $1.21 \pm 1.3$ for adults. Coop and Forrester ${ }^{11}$ compared the pain during skin-prick testing and found that the actual pain experienced was perceived to be much less than the anticipated pain. The post-procedure pain scores were 1.3 to 2.4 points lower than the anticipated pre-procedure pain scores. Children aged 3 to 8 years reported an average pain score of 1.7 , which is similar to the value reported in our study. These comparisons may be useful when counseling parents and children about subcutaneous therapy. Studies investigating the amount of pain associated with venipuncture in children typically report a median facial pain score (FPS) of 4.00 to 7.00 if no prophylactic intervention is applied. Our findings suggest that subcutaneous injections produce less pain than venipuncture. ${ }^{12-14}$ Although the median amount of pain reported in our study is relatively low, it is important to note that some children experienced moderate to severe pain due to the injections. Systematic reviews have reported that distraction is an effective coping method to alleviate needle-related pain in pediatric patients. ${ }^{15,16}$ Another study of adolescents assessed pain during pricking and intradermal allergy skin testing and demonstrated that distractions helped patients to experience less pain. ${ }^{17}$ In this case, injections were given in a friendly, consistent environment and were mostly provided by one pediatric nurse who had had many years of experience giving allergy injections to children. Subcutaneous allergen-specific immunotherapy is generally safe and well-tolerated when used in appropriately selected patients. However, local and systemic reactions may occur.

In our study, only mild local reactions, such as redness or itching at the injection site were occurred. They were managed with local treatment (cool compresses) and oral antihistamines. No systemic reactions, such as anaphylaxis occurred. Additionally, patients who had adverse reactions report greater intensity of pain (median value $=2$ ) compared to patients without local adverse reactions (median value $=1$ ). Rodriguez et al. ${ }^{18}$ conducted a prospective European survey in over 1500 patients to evaluate the safety of allergen immunotherapy (AIT) in the pediatric population (aged 18 or younger). Both SCIT and SLIT were used in $90 \%$ of the pediatric patients to treat single-allergen allergic rhinitis. Of the patients, $1.53 \%$ experienced 29 systemic reactions, with respiratory and skin symptoms being the most common; only three were identified with anaphylaxis, two of which were treated with epinephrine. The risk of systemic reactions was found to be lower in dust mite-sensitized patients than in pollen-allergic patients. 
Our study has several limitations. Due to the observational nature and relatively small size of our study, the number of patients under 8 years of age was quite low. A future analysis with a greater number of children and different injection visits may reveal more robust results. Another limitation is the indirect method used to measure pain. Another possible bias lies in our choice of the subcutaneous route, which is based on the assumption that patients have no reluctance regarding injections. Despite all these limitations, ours is a prospective real-life study of pediatric patients undergoing SCIT. Moreover, our study adds data that we consider relevant to the assessment of the safety associated with this therapy.

\section{Conclusions}

We found a low overall score for the intensity of pain caused by SCITA. Our study confirms that pain should not be an overwhelming limiting factor for performing SCITA in pediatric patients. We also experienced a low number of adverse reactions, thus confirming the safety of SCITA for treating most pediatric patients with allergies.

\section{Authorship}

Authors Cristiana Ferreira and Isabel Rezende were responsible for the conception and design of the study, or acquisition of data, or analysis and interpretation of data. Arminda Guilherme drafted and revised the article critically for important intellectual content. The final approval of the version to be submitted was done by Inês Lopes. All authors read and approved the final manuscript.

\section{References}

1. Dhami S, Nurmatov U, Arasi S, Khan T, Asaria M, Zaman H, et al. Allergen immunotherapy for allergic rhinoconjunctivitis: a systematic review and meta-analysis. Allergy. 2017;72:15971631. https://doi.org/10.1111/all.13201

2. Durham R, Penagos M. Sublingual or subcutaneous immunotherapy for allergic rhinitis? J Allergy Clin Immunol. 2016;137(2):339. e10-349.e10. https://doi.org/10.1016/j.jaci.2015.12.1298

3. Kristiansen M, Dhami S, Netuveli G, Halken S, Muraro A, Roberts $G$, et al. Allergen immunotherapy for the prevention of allergy: a systematic review and meta-analysis. Pediatr Allergy Immunol. 2017;28:18-29. https://doi.org/10.1111/pai.12661
4. Breivik H. International Association for the Study of Pain: update on WHO-IASP activities. J Pain Symptom Manag. 2002;24(2):97-101. https://doi.org/10.1023/A:1013810912609

5. Montalvo A, Martín S, Mesa A, Cortés C, Rodríguez M, Laso MT. Comparative study of 3 types of lancets for performing prick tests. Allergol Immunopathol (Madr). 1996;24:58-64.

6. Abu-Saad $H$, Hamers $H$. Decision-making and paediatric pain: a review. J Adv Nurs. 1997;26(5):946-952. https://doi. org/10.1046/j.1365-2648.1997.00416.x

7. Von Baeyer CL. Children's self-report of pain intensity: what we know, where we are headed. Pain Res Manag. 2009;14:3945. https://doi.org/10.1155/2009/259759

8. Von Baeyer CL. Children's self-reports of pain intensity: scale selection, limitations and interpretation. Pain Res Manag. 2006;11:157-162. https://doi.org/10.1155/2006/197616

9. Sastre MT, Albaret MC, Escursell R, Mullet E. Fear of pain associated with medical procedures and illnesses. Eur J Pain. 2006;10:57-66. https://doi.org/10.1016/j.ejpain.2005.01.009

10. Spínola MA, Cosme J, Fernandes M, Machado C, Resende A, Pereira Barbosa M. Intensity of pain associated with subcutaneous administration of allergen immunotherapy. Rev Port Imunoalergologia. 2018;26:35-46.

11. Coop C, Forester J. Comparison of pain during skin-prick testing, immunizations, and phlebotomy. Allergy Asthma Proc. 2016;37:93-97. https://doi.org/10.2500/aap.2016.37.3961

12. Inal S, Kelleci M. Relief of pain during blood specimen collection in pediatric patients. Am J Matern Child Nurs. 2012;37:339345. https://doi.org/10.1097/NMC.0b013e31825a8aa5

13. Inal S, Kelleci M. Distracting children during blood draw: looking through distraction cards is effective in pain relief of children during blood draw. Int J Nurs Pract. 2012;18:210219. https://doi.org/10.1111/j.1440-172X.2012.02016.x

14. Baxter L, Cohen L, McElvery L, Lawson L, von Baeyer L. An integration of vibration and cold relieves venipuncture pain in a pediatric emergency department. Pediatr Emerg Care. 2011;27:1151-1156. https://doi.org/10.1097/PEC. 0b013e318237ace4

15. Uman S, Birnie A, Noel M, Parker A, Chambers T, McGrath J, et al. Psychological interventions for needle-related procedural pain and distress in children and adolescents. Cochrane Database Syst Rev. 2013;10:CD005179. https://doi.org/10.1002/ 14651858.CD005179.pub3

16. Koller D, Goldman D. Distraction techniques for children undergoing procedures: a critical review of pediatric research. JPediatr Nurs. 2012;27:652-681. https://doi.org/10.1016/j.pedn. 2011.08.001

17. Jeffs A. A pilot study of distraction for adolescents during allergy testing. J Spec Pediatr Nurs. 2007;12:170-185. https:// doi.org/10.1111/j.1478-5153.2007.00220_1.x

18. Rodriguez P, Vidal C, Just J, Tabar Al, Sanchez-Machin I, Eberle $P$, et al. The European survey on adverse systemic reactions in allergen immunotherapy (EASSI): a pediatric assessment. Pediatr Allergy Immunol. 2017;28:60-70. https:// doi.org/10.1111/pai.12660 